\title{
OPEN Disease-related Huntingtin seeding activities in cerebrospinal fluids of Huntington's disease patients
}

\author{
C. Y. Daniel Lee ${ }^{1,2}$, Nan Wang ${ }^{1,2}$, Koning Shen ${ }^{3,10}$, Matthew Stricos ${ }^{1,2}$, Peter Langfelder ${ }^{1,2}$, \\ Kristina H. Cheon ${ }^{1,2}$, Etty P. Cortés ${ }^{4}$, Harry V. Vinters ${ }^{5}$, Jean Paul Vonsattel ${ }^{4}$, \\ Nancy S. Wexler ${ }^{6,7}$, Robert Damoiseaux ${ }^{8,9}$, Judith Frydman ${ }^{3} \&$ X. William Yang ${ }^{1,2} \bowtie$
}

In Huntington's disease (HD), the mutant Huntingtin (mHTT) is postulated to mediate templatebased aggregation that can propagate across cells. It has been difficult to quantitatively detect such pathological seeding activities in patient biosamples, e.g. cerebrospinal fluids (CSF), and study their correlation with the disease manifestation. Here we developed a cell line expressing a domainengineered mHTT-exon 1 reporter, which showed remarkably high sensitivity and specificity in detecting $\mathrm{mHTT}$ seeding species in HD patient biosamples. We showed that the seeding-competent mHTT species in HD CSF are significantly elevated upon disease onset and with the progression of neuropathological grades. Mechanistically, we showed that mHTT seeding activities in patient CSF could be ameliorated by the overexpression of chaperone DNAJB 6 and by antibodies against the polyproline domain of mHTT. Together, our study developed a selective and scalable cell-based tool to investigate $\mathrm{mHTT}$ seeding activities in HD CSF, and demonstrated that the CSF mHTT seeding species are significantly associated with certain disease states. This seeding activity can be ameliorated by targeting specific domain or proteostatic pathway of $\mathrm{mHTT}$, providing novel insights into such pathological activities.

Huntington's disease (HD) is a dominantly inherited neurodegenerative disorder caused by CAG repeat expansion encoding a polyglutamine (polyQ) stretch near the N-terminus of mutant Huntingtin (mHTT). The mutant polyQ repeat increases the propensity of $\mathrm{mHTT}$ to misfold and form aggregates in the striatum and cortex of HD patients ${ }^{1}$. The precise pathogenic roles of the mHTT aggregates remain elusive. Certain studies suggest that they may be protective ${ }^{2}$ or neutral ${ }^{3,4}$, while others show that they are pathogenic ${ }^{5-7}$ and possibly contribute to the selective vulnerability of striatal medium spiny neurons (MSNs) in $\mathrm{HD}^{8}$.

Several antibody-based platforms have been developed to quantitatively detect HTT species as a biomarker in biosamples ${ }^{9,10}$. The Singulex single-molecule counting immunoassay (SMC) can detect a significant increase of HTT in cerebrospinal fluids (CSF) and plasma from premanifest to manifest HD stages ${ }^{9,10}$. Importantly, this assay has been successfully employed in the antisense oligonucleotide (ASO) trial in HD patients to show the reduction of $\mathrm{mHTT}$ in the CSF of HD patients ${ }^{11}$. The ELISA-based meso-scale discovery (MSD) electrochemiluminescence assay is a platform that sensitively quantifies and differentiates soluble and aggregated mHTT in brain tissues and blood cells ${ }^{12-14}$. The microbead-based immunoprecipitation and flow cytometry assay (IP-FCM) has been shown to detect mHTT in the brain of HD mouse models and CSF of patients ${ }^{15}$. The study further showed that ASO against HTT could reduce CSF mHTT in BACHD, a mouse model of HD ${ }^{15,16}$. These sensitive antibody-based methods demonstrated the presence of mHTT in the more accessible patient biofluids. However, since HTT is a large protein $(350 \mathrm{kDa})$ with extensive post-translational modifications and aggregates to

\footnotetext{
${ }^{1}$ Center for Neurobehavioral Genetics, The Jane and Terry Semel Institute for Neuroscience \& Human Behavior, University of California, Los Angeles, Los Angeles, USA. ${ }^{2}$ Department of Psychiatry and Biobehavioral Sciences, David Geffen School of Medicine at UCLA, Los Angeles, CA, USA. ${ }^{3}$ Department of Biology and BioX Program, Stanford University, Stanford, CA, USA. ${ }^{4}$ Division of Aging and Dementia, Department of Neurology, Columbia University Medical Center, New York, NY, USA. ${ }^{5}$ Department of Pathology and Laboratory Medicine, Neurology, David Geffen School of Medicine at UCLA, Los Angeles, CA, USA. ${ }^{6}$ Departments of Neurology and Psychiatry, College of Physicians and Surgeons, Columbia University, New York, NY, USA. ${ }^{7}$ Hereditary Disease Foundation, New York, NY, USA. ${ }^{8}$ California NanoSystems Institute, University of California, Los Angeles, CA, USA. ${ }^{9}$ Department of Molecular and Medical Pharmacology, University of California, Los Angeles, CA, USA. ${ }^{10}$ Present address: Department of Molecular and Cell Biology, UC Berkeley, Berkeley, CA, USA. ${ }^{\circledR}$ email: xwyang@mednet.ucla.edu
} 
form higher order structures ${ }^{17,18}$, it is likely that these antibody-based assays may capture a subset (e.g. specific conformations) of mHTT species.

Emerging studies show that pathogenic proteins in a variety of neurodegenerative disorders may exhibit "prion-like" properties, leading to the spreading and template-based amplification of misfolded disease proteins (e.g. aggregated a-synuclein in Parkinson's disease and hyperphosphorylated Tau in Tauopathy and Alzheimer's disease $)^{19,20}$. This is postulated to be a key mechanism in disease progression ${ }^{19,20}$. Previous studies reported that the aggregation of recombinant mHTT fragments in vitro could be greatly accelerated by the addition of preformed mHTT aggregates ${ }^{21}$, a phenomenon often referred to as mHTT "seeding activities". Moreover, extracellular application of preformed mHTT aggregates or crude extracts of aggregates from postmortem HD brains or mouse models of HD could also seed intracellular aggregation of otherwise soluble forms of $\mathrm{HTT}^{22-25}$. One later study demonstrated that extracts from modified PC12 cells expressing inducible GFP-tagged mHTT-exon 1 fragment with 103Q showed increased aggregation with the addition of CSF from HD patient ${ }^{26}$. It sheds the light of using seed-induced aggregation for detecting mHTT seeding species in the biosamples. However, this assay is limited by its sensitivity and scalability since the measurements of induced aggregation rely on cell counting and filter retardation assays, and the cell line used in this study spontaneously forms reporter protein aggregates without the addition of extracellular mHTT seeds ${ }^{26}$.

In this study, we developed cell lines stably expressing wildtype and engineered mHTT-exon 1 reporters. We found that deleting the N17 domain of mHTT-exon 1 greatly augments the sensitivity of the reporter cells for detection mHTT seeding species. Importantly, with CSF samples from postmortem brains and living HD patients, we showed that the seedable mHTT species in the patient CSF increased with the progression of clinical disease presentation and neuropathology. Moreover, we showed the CSF mHTT seeding activities can be abolished by overexpressing the chaperone DNAJB6 and by antibodies against the polyproline domain of HTT. These results suggest the presence and level of seeding-competent mHTT species in the patient CSF may correlate with neuropathological progression, and substantiate the use of this highly sensitive and scalable cell-based assay for studying mHTT seeding activities in HD clinical progression and response to therapy.

\section{Results}

Deleting the $\mathrm{N} 17$ domain of $\mathrm{mHTT}$ significantly enhanced the sensitivity of the reporter cells to detect mHTT seeding species. Prior studies showed that reporter cells expressing fluorescent proteins tagged mHTT fragment with either an expanded pathogenic repeat or a short nonpathogenic repeat could be used as intracellular reporters for exogenous "mHTT seeds" induced aggregation ${ }^{22,26}$. However, these cell lines fall short of sensitivity, specificity and scalability in detecting the presence of mHTT seeds in HD patient biosamples, such as CSF. To develop a reporter cell line that fulfill these requirements, we reasoned that the cells should stably express a mHTT N-terminal fragment with a moderately expanded polyQ repeat, which thus could be nucleated to form aggregates while not spontaneously aggregates at the baseline. We first generated a stable HEK293 cell line expressing mHTT-exon 1 fragment with 46Q and in-frame C-terminal green fluorescent protein fusion (mHTT-46Q-GFP; Fig. 1a). We chose this modestly expanded polyQ repeat, which is within the range of the common adult-onset $\mathrm{HD}$ patients ${ }^{27}$. It is metastable and can form oligomers or small aggregates in neurons. Unlike mHTT-exon 1 expression constructs with over $100 \mathrm{Q}^{2,28}$, mHTT-46Q peptides rarely form aggregates when expressed at a moderate level in cultured cells ${ }^{29}$. Consistent with this observation, we found that HEK293 cells stably expressing mHTT-46Q-GFP did not show visible aggregates at the baseline (Fig. 1c). We treated this cell line with purified recombinant mHTT-exon 1 with 51Q (mHTT-51Q) in the forms of monomers, fibrils and sonicated fibrils (also referred as mHTT seeds hereafter; $\Phi=30.7-49.6 \mathrm{~nm} ;$ Fig. $1 \mathrm{~b})^{30}$. We found increasing levels of $\mathrm{GFP}^{+}$aggregates in reporter cells treated with extracellular mHTT fibrils and seeds, while monomeric mHTT-51Q did not induce mHTT-46Q-GFP aggregation (Fig. 1c).

Since the first 17 amino acid (N17) of mHTT is critical in regulating the subcellular localization, oligomerization and aggregation, and toxicities of mHTT in vitro and in vivo ${ }^{29-31}$, we tested whether mutations in the N17 domain influenced the sensitivity of the reporter cells in detecting extracellular mHTT fibrils and seeds. To this end, we developed cell lines stably expressing mHTT-exon 1-46Q-GFP reporters with two distinct N17 mutations (Fig. 1a), i.e. the S13D/S16D mutations that block mHTT oligomerization and aggregation in vitro and in vivo ${ }^{32,33}$, and the $\Delta \mathrm{N} 17$ mutation (a deletion of amino acid 2-16) that leads to increased oligomer formation, nuclear mHTT aggregation and HD-like disease phenotypes in vivo ${ }^{29,30,34}$. These two cell lines are termed mHTTSD-GFP and mHTT- $\triangle$ N17-GFP, respectively. Similar to the mHTT-46Q-GFP cell line, both mHTT-SD-GFP and mHTT- $\triangle$ N17-GFP cell lines have neglectable aggregation at baseline and when treated with mHTT-51Q monomers (Fig. 1c,d). Interestingly, these two new cell lines showed distinct effects when treated with the mHTT fibrils and seeds. The mHTT- $\triangle$ N17-GFP cells showed a dramatic enhancement of mHTT-51Q fibrils- and seedinduced aggregation, while mHTT-SD-GFP cells showed comparable or possibly modest reduction of those induced aggregation compared to the mHTT-46Q-GFP cell line (Fig. 1c,d). Moreover, we found the mHTT-51Q seed-treated cells show more $\mathrm{GFP}^{+}$aggregates compared to the mHTT-51Q fibril treated cells (Fig. 1d,e). Notably, while the size range of the aggregates is comparable between the two treatment (mostly between 0.3 and $2 \mu \mathrm{m}$ ), the mHTT-51Q seeds tend to induce a greater number of smaller-sized aggregates than the fibrils did (Fig. 1e and Supplemental Fig. S1). Together, we showed that deletion of N17 from mHTT-46Q-GFP helped to generate a highly sensitive cell line in detecting the exogenous mHTT fibrils and seeds, while maintaining minimal spontaneous aggregation of the reporter proteins at the baseline.

Deletion of N17 domain in the mHTT-46Q-GFP reporters is necessary for enabling detection of $\mathrm{mHTT}$ seeding activities in HD brain samples. A litmus test for any disease protein seeding assay is whether it can reliably detect the aggregate-inducing, disease-specific protein species in the biosamples. We first 


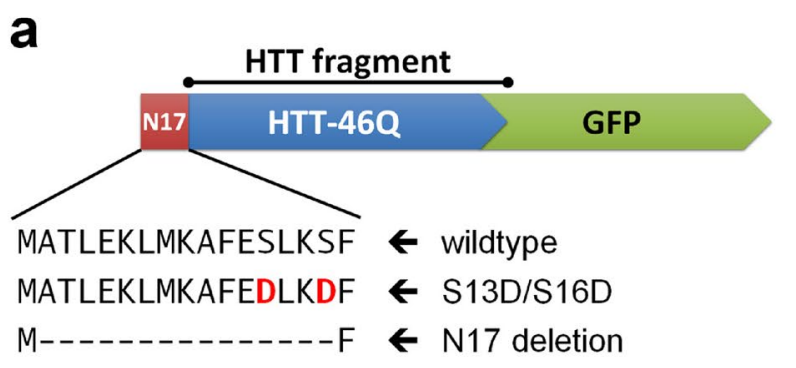

b
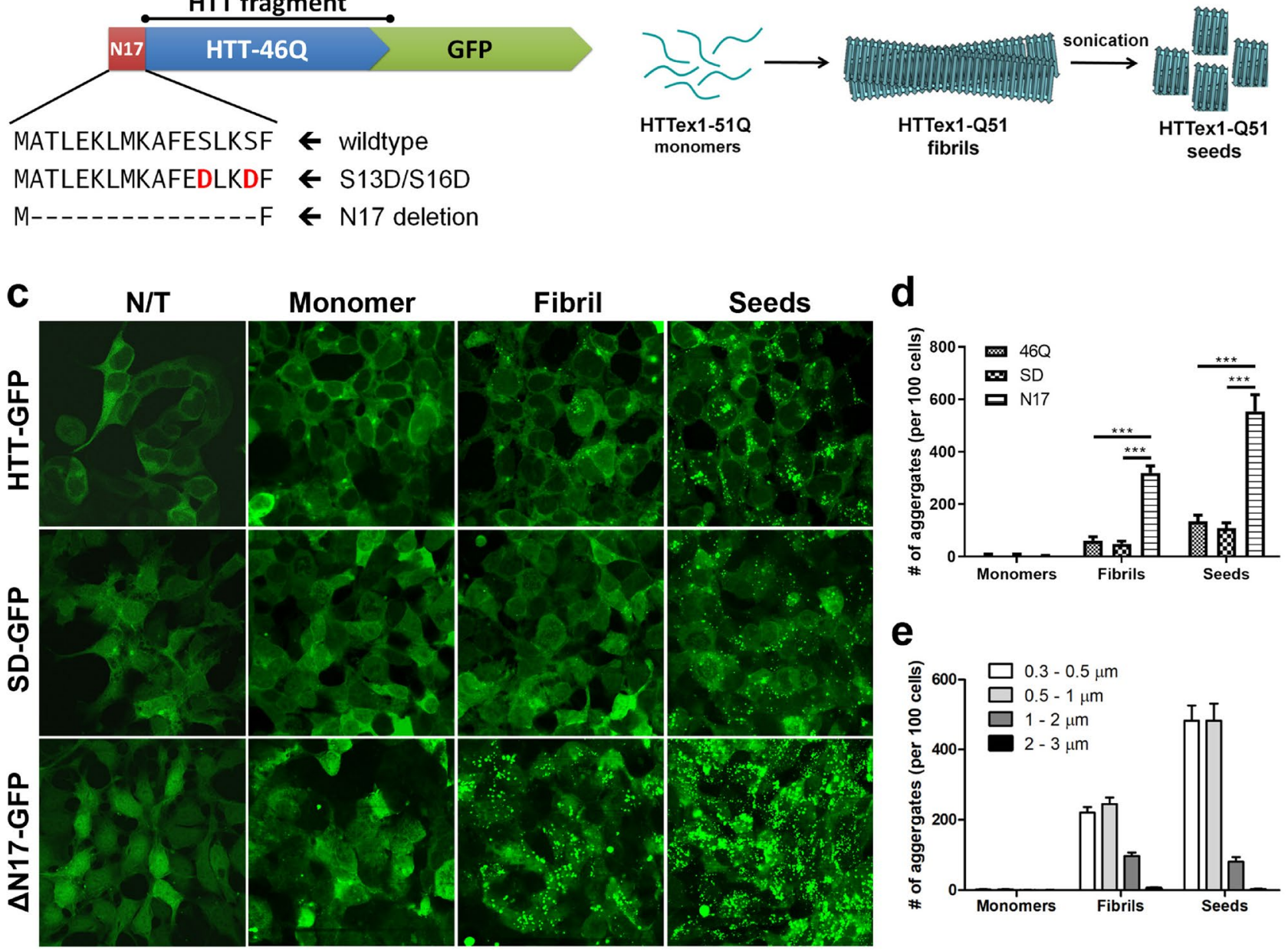

Figure 1. N17 mutations in recipient cell lines altered sensitivity of seeding-induced mHTT aggregation. (a) A diagram illustrates the constructs of GFP-tagged mHTT-exon 1 (46Q) fragments, which are used to generate stable cell lines. (b) A diagram illustrates the generation of mHTT aggregates (fibrils) and seeds from monomeric recombinant mHTT-51Q proteins. (c) Cell lines generated by stably transfected HEK-293 cells with GFP tagged mHTT-exon 1 (46Q) variants were used as recipient cells of the seeding assay. Constructs of the transgenes are labeled on the left of the panel. Recombinant mHTT-exon 1 (51Q) in the forms of monomers, fibrils and seeds were added directly to the cultured cells for 3 days. Representative confocal images of the cells are shown. Bright green punctae are intracellular mHTT-GFP aggregates induced by seeding. N/T = nontreated. (d,e) The number of HTT-GFP aggregates was quantified using ImageJ and presented as mean \pm s.e.m., $n=5-10,{ }^{* *} p<0.01$.

tested our three reporter cell lines using brain homogenates from the Q175 mice, a full-length murine mutant Huntingtin (mHtt) knock-in mouse model carrying an expanded CAG repeat and showing early and progressive $\mathrm{mHtt}$ aggregation, transcriptional dysregulation, and behavioral impairment (Fig. 2) ${ }^{35-38}$. Among the three reporter cell lines, only the $\mathrm{mHTT}-\triangle \mathrm{N} 17-\mathrm{GFP}$ line is sensitive enough to show induced aggregation with sonicated striatal extracts from old (14 months of age) but not young (4 months of age) Q175 mice (Fig. 2o,p), nor with those from aged wildtype mice (Fig. 2n). Importantly, when applying sonicated cortical and striatal lysates from postmortem HD patients, we also detected GFP ${ }^{+}$aggregates only in mHTT- $\Delta$ N17-GFP cells but not in the other cell lines (Fig. 2). These results demonstrate that only the reporter cells expressing the $\Delta \mathrm{N} 17$ version of mHTT-46Q-GFP, but not the unmodified or SD versions, have the sensitivity to detect mHTT seeding activities of the brain samples. The study with Q175 striatal extracts also suggests that the seeding activities detected by the mHTT- $\triangle$ N17-GFP cells reflect specific age-dependent mHTT species emerged with disease progression, not just due to the expression of mHTT.

Quantitative and scalable analyses of mHTT seeding species using the mHTT- $\Delta$ N17-GFP reporter cell line. To further enhance the quantification and high-throughput capability, we adapted the assay into a 384-well platform and utilized a high-content imaging system to quantify seed-induced reporter aggregation. We first compared the measurement of time-dependent aggregation using manual counting versus using automated detection after seeding (Fig. 3a,b). Both methods were capable of capturing the increase of induced $\mathrm{GFP}^{+}$aggregates over time. We noticed that the smaller aggregates $(<0.9 \mu \mathrm{m})$ were not detected reli- 


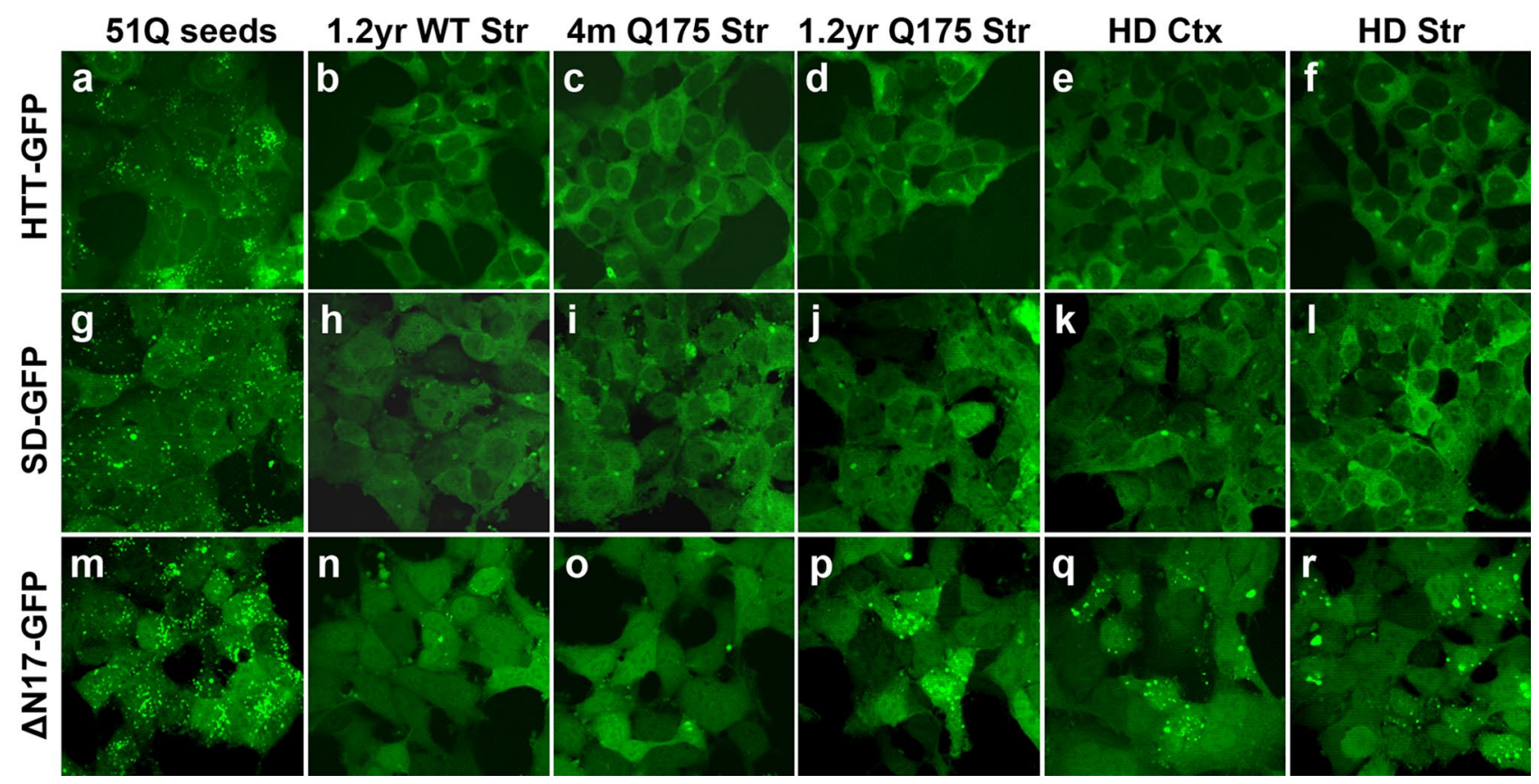

Figure 2. Deletion of $\mathrm{N} 17$ enables detection of mHTT seeding activities in HD mouse and patient brain lysates. Recombinant mHTT-51Q seeds and brain lysates from HD patients and mouse models were applied directly to mHTT-46Q-GFP (a-f), mHTT-SD-GFP $(\mathbf{g}-\mathbf{l})$ or mHTT- $\Delta$ N17-GFP cell lines $(\mathbf{m}-\mathbf{r})$ for 3 days. Representative confocal images of the cells are shown.

ably by the automated image analysis, possibly due to the optical limitation and background heat noise from the instrument. Thus, for optimal signal detection over the background, we chose to only quantify the GFP ${ }^{+}$ aggregates at the size between 1 and $3 \mu \mathrm{m}$. As a result, the 384-well format and the high-content imaging system were capable of capturing the time-dependent progression of mHTT- $\Delta$ N17-GFP (referred to as mHTT-GFP thereafter) aggregation (Fig. 3b). Additionally, the level of aggregation reflected the amount of recombinant mHTT-51Q seeds applied as a function of saturation curve (Fig. 3c). Since the concentration of $2 \mu \mathrm{g} / \mathrm{ml}$ of extracellular mHTT-51Q seeds is well within the linear range of the dose-response curve, we used this fixed amount of mHTT-51Q seeds $(2 \mu \mathrm{g} / \mathrm{ml})$ as a positive control and for normalizing the aggregation readouts in all subsequent studies. We operationally defined an aggregation index of 100 arbitrary units as the number of mHTT-GFP aggregates induced by exogenous addition of $2 \mu \mathrm{g} / \mathrm{ml}$ of mHTT-51Q seeds onto the mHTT- $\Delta$ N17GFP cells in the 384-well format for all later experiments.

The mHTT- $\Delta$ N17-GFP cell line is specific for detecting mHTT seeding activities originated from recombinant $\mathrm{mHTT}$ seeds and HD patient samples. To evaluate the specificity of the mHTTGFP aggregation in response to the exogenous seeds, we applied sonicated fibrillar $A \beta$ and hyperphosphorylated Tau aggregates to the mHTT- $\Delta$ N17-GFP cells. Neither of these protein aggregates induced mHTT- $\Delta$ N17-GFP aggregation in the reporter cells (Fig. 3d and Supplemental Fig. S2). We further explored whether the seedingcompetent (or "seedable") mHTT species exist in the postmortem CSF samples, i.e. the mHTT seeding activities, from HD and other neurodegenerative disease patients (UCLA's neuropathological collection; see methods). Application of postmortem CSF from HD patients significantly increased GFP ${ }^{+}$aggregates in mHTT- $\Delta$ N17GFP cells. On the contrary, such induced aggregation was not found when the cells were treated with postmortem CSF from Alzheimer's disease (AD) or Parkinson's disease (PD) patients (Fig. 3e, Supplemental Fig. S2 and Table S1). Together, our results suggest that the seed-induced mHTT-GFP aggregation in mHTT- $\Delta$ N17-GFP cells is highly specific to recombinant mHTT aggregates, and to the seeding-competent species present in the CSF of HD patients.

The mHTT seeding activities in the postmortem CSF of the HD patients are correlated with neuropathological grades of the brains. We next investigated whether the levels of CSF mHTT seeding activities correlate with the pathological progression in the brain, which was measured by Vonsattel neuropathological grades, a postmortem evaluation of neuronal cell loss in the caudate and putamen of HD patients ${ }^{39}$. We assayed a panel of autopsy CSF samples with HD neuropathological staging performed by Dr. Vonsattel at Columbia University Medical Center (Supplemental Table S2). The autopsy CSF from HD Stage 3 and Stage 4 subjects (NY Brain Bank or NYBB cohort) induced significantly more mHTT-GFP aggregation compared to controls, while CSF from HD1 and HD2 patients did not significantly increase aggregation in these cells (Fig. 4a). The statistical significance of the latter groups may be in part limited by the small sample size $(n=3$ for HD1 and HD2 combined) (Fig. 4a). Importantly, CSF from HD4 subjects also induced significantly more mHTT-GFP aggregation than those from HD1 and HD2 patients, suggesting an HD neuropathological stage- 

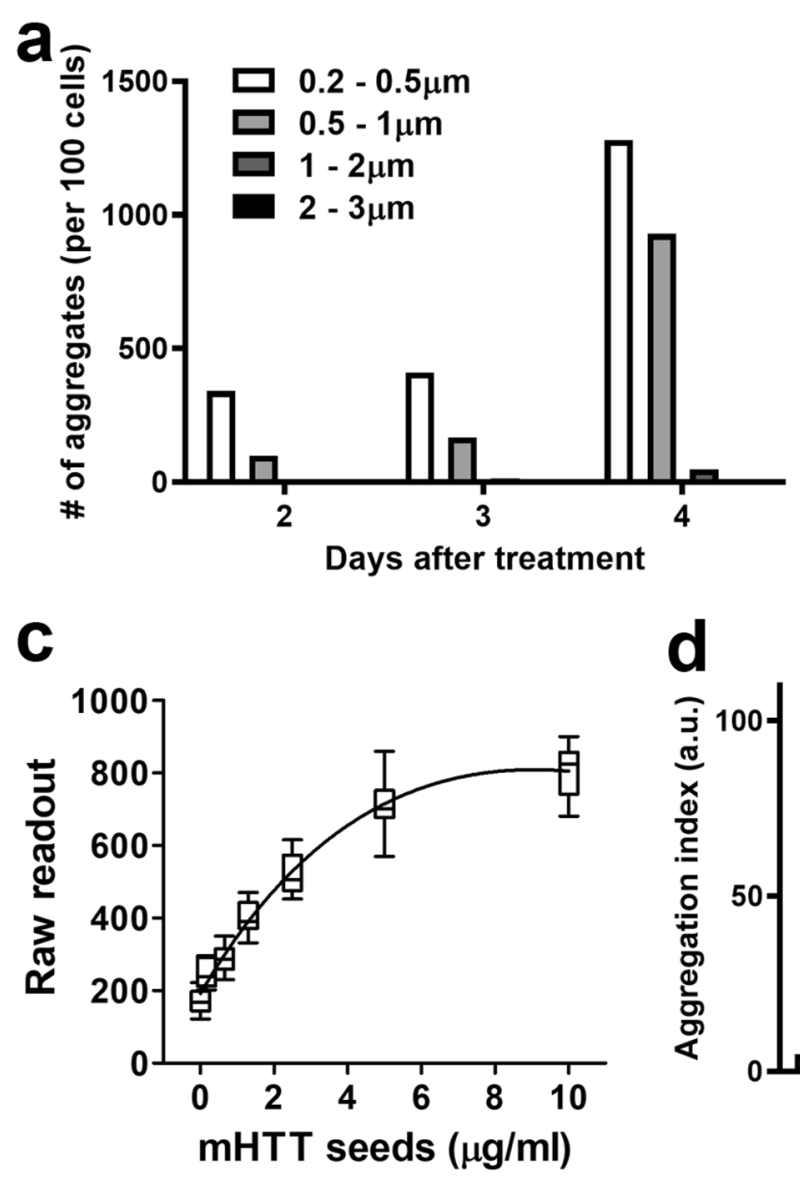

b

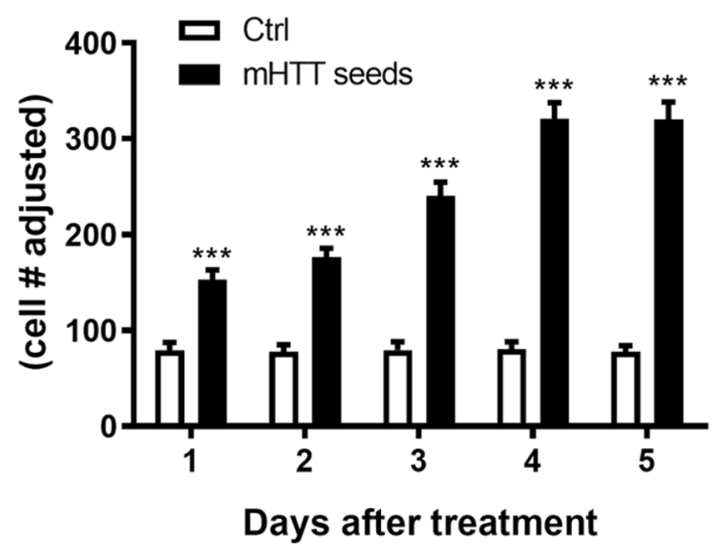

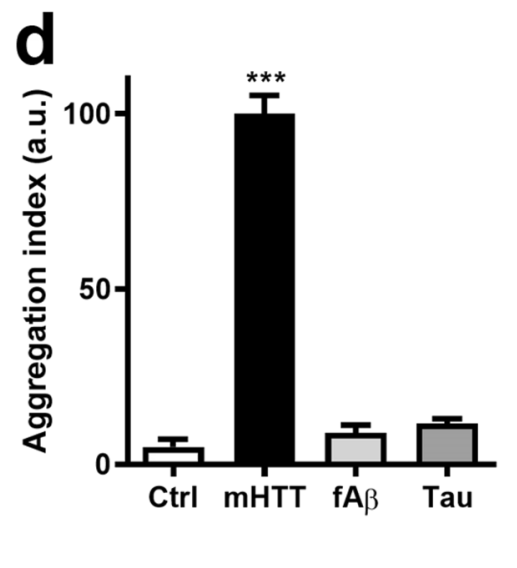

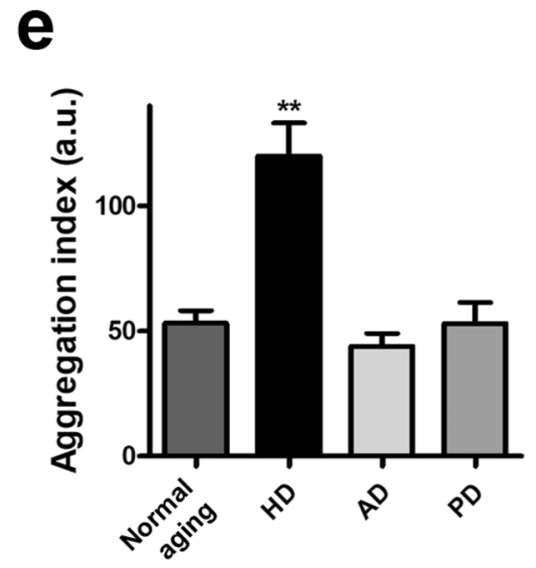

Figure 3. The seeding assay using mHTT- $\triangle$ N17-GFP cells is scalable and could specifically and sensitively detect mHTT seeding activity in the biosamples of HD patients. (a,b) Recombinant mHTT seeds were applied to mHTT- $\triangle$ N17-GFP cells in 24-well (a) and 384-well (b) settings for indicated duration of time $(n=6-8)$. (c) $\mathrm{mHTT}$ at a range of concentrations were applied to mHTT- $\triangle$ N17-GFP cells for 5 days to generate the dose-response curve ( $\mathrm{n}=6$ for each condition). $(\mathbf{d}, \mathbf{e})$ mHTT- $\Delta$ N17-GFP cells were treated with sonicated recombinant protein aggregates (d; $\mathrm{mHTT}$ seed at $2 \mu \mathrm{g} / \mathrm{ml}$, fA $\beta$ and pTau at $10 \mu \mathrm{g} / \mathrm{ml}$ ) or postmortem CSF from $\mathrm{HD}, \mathrm{AD}$ or PD patients $(\mathbf{e})$ in the 384-well format for 5 days $(n=6-8)$. Confocal images were acquired manually on a Zeiss LSM510 confocal microscope and analyzed with ImageJ (a), or captured with automation on an ImageXpress Micro Confocal and analyzed with MetaXpress $(\mathbf{b}-\mathbf{e})$, respectively. The data are presented as mean \pm s.e.m., ${ }^{* *} p<0.01 ;{ }^{* *} p<0.001$.

dependent increase of seeding-competent mHTT species in the CSF. To evaluate possible confounding factors, we tested linear models of the aggregation index regressed on HD grade (treated as a categorical variable) and potential confounders including age, PMI and gender (Supplemental Table S4). We found that the association between HD4 and the aggregation index remained significant $(p=0.02)$. We further test the all combination of these covariates plus CAG length. HD grades regressed on the aggregation index remains the best model, since addition of other covariates was more likely deteriorate the fit (Supplemental Table S4). Analyzing the confounders individually, the age and CAG length of subjects are not correlated with the seedability of HD CSF (Fig. 4b,c). We also found no correlation between PMI and the seeding activities of the CSF (Fig. 4d). Thus, the strong correlation between the level of seeding-competent mHTT species in CSF and HD pathology suggests that these mHTT species in CSF, which could be detected by the mHTT- $\triangle$ N17-GFP reporter cell assay, might elevate as neuropathology progressing in the brain.

Preliminary analysis of mHTT seeding activities in CSF of living HD patients suggests its association with disease progression. Postmortem pathological grades indicate the end stage condition of the brain. However, whether the pathological measurement could be related to clinical severity of HD remains debatable. We began to address this question by investigating whether our assay could detect mHTT seeding species in the CSF from living HD gene carriers and patients, and if such study could provide initial insights on the role of seeding activities with the disease. The CSF samples were acquired from the PREDICT-HD collection (NINDS BioSEND), a longitudinal phenotypic and biomarker study of prodromal HD (see "Methods" section and Supplemental Table S3) ${ }^{40}$. In PREDICT-HD, the enrolled individuals were evaluated annually for motor and cognitive functions, performed brain imaging and collected biosamples. They are categorized by the diagnostic confidence level (DCL), where DCL0 means indistinguishable from healthy individuals, DCL2 and 

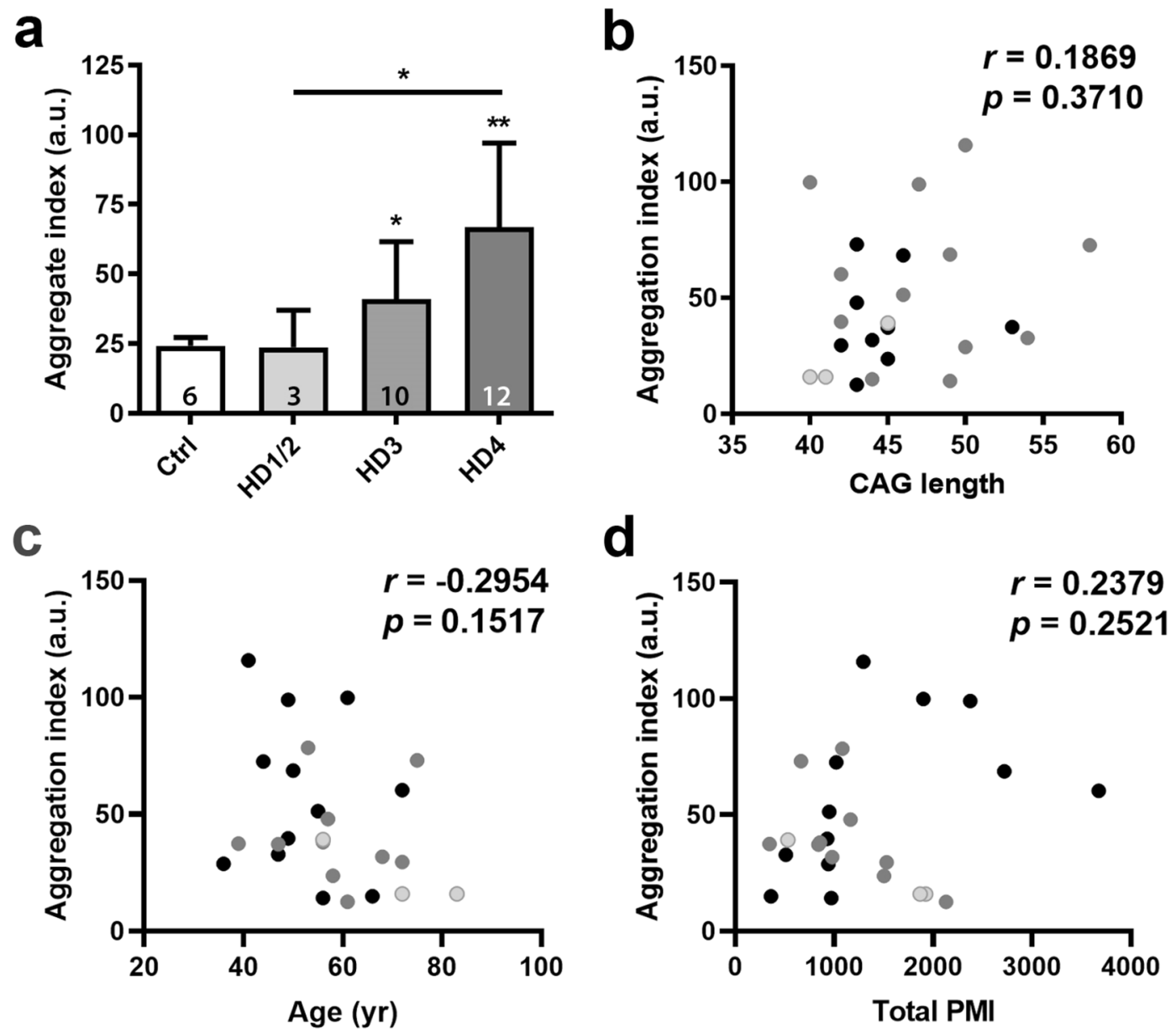

HD1/2 HD3 $\bullet \mathrm{HD} 4$

Figure 4. The mHTT seeding activities in the postmortem CSF is correlated with neuropathological grade based on striatal degeneration. The disease status of the patients were graded based on their neuropathological features. Postmortem CSF samples from these subjects and non-HD controls were applied to the mHTT- $\Delta$ N17GFP cells at 1:20 dilution in the 384-well format for 5 days. Confocal images were acquired on ImageXpress Micro Confocal and used to measure induced mHTT-GFP aggregation using MetaXpress. (a) CSF samples were grouped based on their pathological grading. The number of samples is as shown in the bar. HD1 and HD2 samples were grouped together for statistical analysis. One-way ANOVA with Tukey post-hoc analysis was performed to determine the $p$ value versus the non-HD controls, or otherwise as indicated. Bar graphs are shown as mean \pm s.d. ${ }^{*} p<0.05,{ }^{* *} p<0.01$. (b-d) Correlation analysis between the CSF seeding activities and the CAG repeat length $(\mathbf{b})$, the age of the HD patients (c) or PMI of the CSF samples (d). Individual dots are color coded by their neuropathological scores. Pearson's correlation analysis was performed to determine the Pearson's $r$ and $p$ value.

DCL3 are prodromal stages with increasing likelihood of HD diagnosis, and DCL4 is most likely to be clinically diagnosed HD. To prevent experimental bias, we were provided 60 PREDICT-CSF samples by NINDS/BioSEND and performed the seeding assays while blinded to any identification or clinical information. Samples were unblinded only after the results were submitted back to NINDS BioSEND. We found that the 60 CSF samples were actually duplicates of 30 samples collected from 2 visits of 15 individuals (including controls, mHTT gene carriers and HD patients; Supplemental Table S3). This experimental design was aimed to evaluate incurred sample reproducibility of our assay. The results demonstrated satisfying high incurred sample reproducibility (Pearson's $r=0.9481, p<0.0001$; Fig. 5a). We found a significant increase in the mHTT seeding activities of the CSF only from the DCL4 group, but not from the pre-HD (DCL1-DCL3) subjects, even though they already displayed some signs of motor abnormalities (Fig. 5b). To account for correlation of aggregation index measurements of the two visits for each subject, we used linear mixed model with the variable of interest (DCL score) as a categorical fixed effect and the subject as a random effect. Here, non-carrier subjects and the DCL0 HD carriers were combined as the control group of healthy individuals to improve statistical power. We found significant association for DCL4 versus DCL0 (healthy individual group) in the pairwise comparison and for DCL4 versus the rest groups in the full model (Supplemental Table S5). To ensure that this result is not due to confounding, we carried out a comprehensive study of regression with all combination of the following 8 covariates: age, sex, 


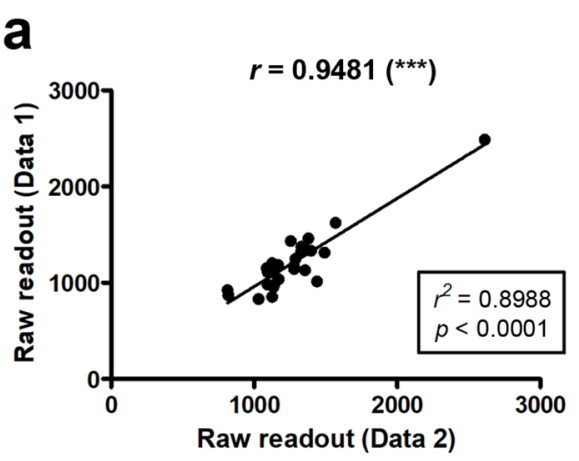

d

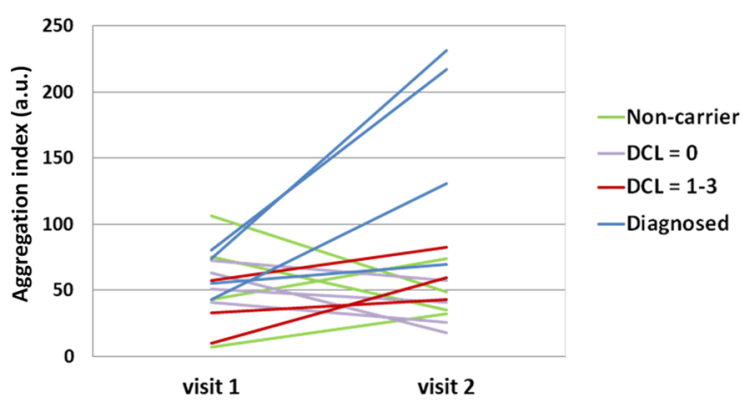

b $\quad$ c

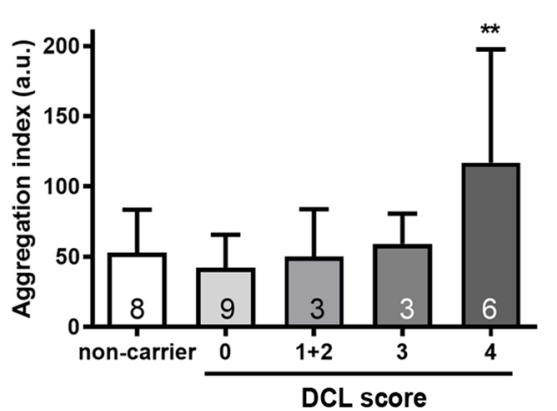

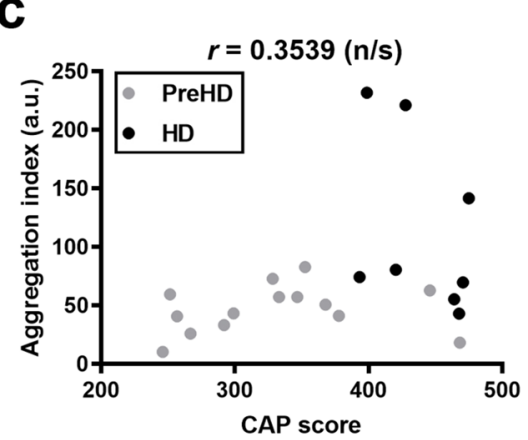

Figure 5. High reproducibility of detecting disease-related mHTT seeding activities in CSF from living HD patients. (a) Identity blinded CSF samples from the PREDICT-HD collection were provided by BioSEND and applied to the mHTT- $\Delta$ N17-GFP cells at 1:20 dilution in the 384-well format for 5 days. Confocal images were acquired with automation and batch analyzed for aggregation scores. Upon unblinding the samples, the two aggregation readouts from the duplicated aliquots of the same CSF samples were plotted and showed high reproducibility between the duplicates of the samples using this assay. (b) The HD patients, mutation carriers and non-carrier controls were grouped based on their DCL scores. DCL1 and DCL2 are grouped together due to their small sample sizes. Numbers in the bars indicate the $n$ of each group. (c) The correlation analysis of the CSF seeding activities and the CAP score. (d) The measured aggregation indices of both visits from the same individuals were plotted to visualize the change of CSF ceding activity. (e) The total motor scores was plotted against the CSF mHTT seeding activity to analyze their association. Pearson's correlation coefficients $(r)$ for $(\mathbf{c})$ and (e) were calculated and noted on the top of the plots. Statistical significance ( $p$ value) of the correlation is shown in parentheses. Additional linear regression analyses were performed for $(\mathbf{a}, \mathbf{e})$, and shown in the squares. Bar graphs are presented as mean \pm s.d. ${ }^{* *} p<0.01 ; \mathrm{n} / \mathrm{s}=$ not significant.

education years, height and BMI, CAG (high), CAG (low) and CAP score (there are $2^{8}=256$ combinations). When the covariates are added individually, the association between aggregation score and DCL4 remained significant (Supplemental Table S6). None of the covariates is significantly $(p<0.05)$ associated with aggregation index in any of the models. There are combinations of covariates (e.g., all 8) with which the association with DCL becomes non-significant, likely because these combinations of covariates explain a large fraction (mostly over $50 \%$ ) of the DCL score variation. As a diagnostic check, especially for the small PREDICT-HD sample size, we tested normality of the residuals of aggregation index using Shapiro-Wilks test and found non-significant results (Supplemental Table S7).

Another interesting finding is the lack of significant correlation between mHTT seeding activities and the CAG-age product (CAP) score (Pearson's $r=0.3539$; Fig. $5 \mathrm{c}$ ), a mathematical predictor of HD progression ${ }^{41}$, and CAG repeat length (Pearson's $r=0.09349$; Supplementary Fig. 3b). We also found no significant associations with CAP and CAG using linear mixed effects models (Supplemental Table S5). This finding suggests that our seeding assay is not tracking the CAP score, and also differentiates our seeding assay from the clinically used SMC assay, which is highly correlated with the CAP $\operatorname{scor}^{10}$. Notwithstanding the need to include additional samples to further test the robustness of our seeding assay in clinical samples, our findings thus far suggest that the mHTT seeding activities may be significantly increased upon HD diagnosis and potentially track aspects of the disease that are not measured by the traditional CAP scores or SMC assay.

Further analysis focused on the comparison of the CSF samples collected from two visits, 1 year apart, of the same individuals. This analysis should be considered preliminary due to the limited number of multi-visit CSF samples available from PREDICT-HD. Despite such limitation, we found a significant increase in the change of the CSF seeding activities ( $\Delta$ aggregation index) in individuals diagnosed with HD but not in non-carriers or HD gene carriers in the DCL0 to DCL3 categories (Fig. 5d and Supplemental Fig. S3a). These preliminary results suggest that the level of mHTT seeding species in CSF is significantly progressive only after the subjects are diagnosed. 
a

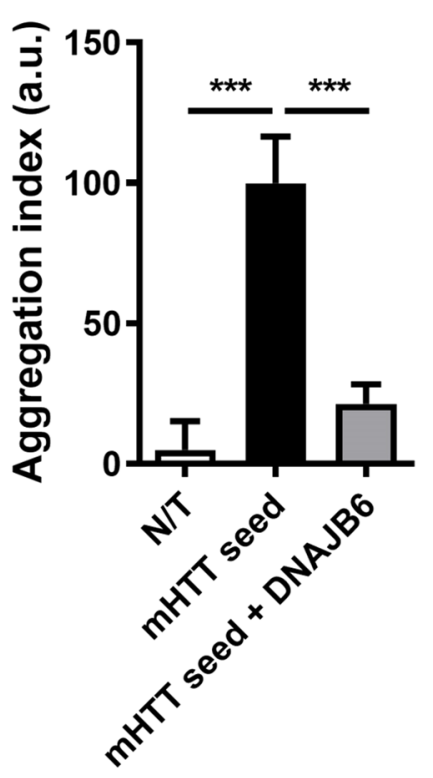

b

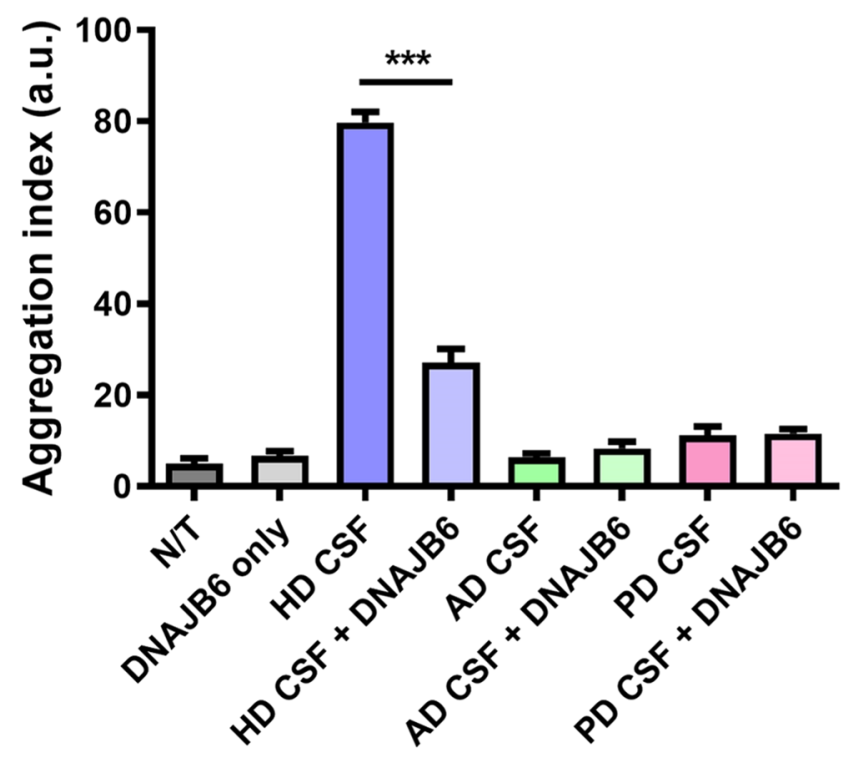

Figure 6. Chaperone DNAJB6 blocks the seeding activities of HD patient CSF. The mHTT- $\triangle$ N17-GFP cells were transfected with DNAJB6 1 day prior to the application of mHTT51Q seeds $(2 \mu \mathrm{g} / \mathrm{ml}$, a) or postmortem CSF from HD, AD and PD patients (1:20, b). The assays were performed in the 384-well format. Confocal images were acquired with automation 5 days after seeding and batch analyzed to calculate Aggregation indices of the samples. Bar graphs are presented as mean \pm s.e.m., $n=6-8,{ }^{* * *} p<0.001, \mathrm{~N} / \mathrm{T}=$ non-treated controls.

We next performed a correlation analysis of the aggregation index with a series of clinical scores, assessed at the time of visits. Again, while these analyses should be considered preliminary, we found a significant correlation between the CSF seeding activity and the disease severity. Increasing seeding propensity of the CSF was significantly correlated with the UHDRS total motor score (Pearson's $r=0.5849, p=0.0054$; Fig. 5e) and total function capacity (TFC; Pearson's $r=-0.7476, p<0.0001$; Supplemental Fig. S3 and Table S3) ${ }^{41}$. We again used mixed effect models to test these associations while accounting for repeat visits and found that the associations of aggregation index and TFC remain significant even after Bonferroni correction for the 69 tested clinical variables (Supplemental Table S7). In contrast, we did not find that the CSF seeding propensity significantly correlate with cognitive tests (e.g. Stroop color naming and word reading scores), while some of these scores are significantly higher in PreHD than manifest HD subjects, nor with the volume changes in cortical and striatal brain regions (Supplemental Fig. S3 and Table S3 and S8).

Together, our study suggests that the mHTT seeding activities in CSF appear to significantly increase as HD diagnosis and are significantly correlated with clinical severity as the disease progress in the subjects. A limitation of our current study is the relatively small number of longitudinal CSF samples available from manifest HD patients with clinical data, and therefore it should be considered preliminary findings. Future longitudinal studies with a large cohort of premanifest and manifest HD patients will be needed to rigorously evaluate the relationships between CSF mHTT seeding activities and disease onset and progression in HD.

Blocking HD CSF mHTT seeding activities with chaperone DNAJB6 and antibodies against the polyproline domain of HTT. We next sought to apply our cell-based mHTT seeding assay to evaluate genes and molecules that block the pathological seeding activities in HD patient CSF, and hence to gain insights into the molecular bases of such disease-related mHTT seeding activities.

DNAJB6 is a member of the Hsp40 chaperone protein family and has been shown to delay polyglutamine aggregation through inhibition of early nucleation and the subsequent amyloid fibril formation ${ }^{42,43}$. We investigated if recombinant seed- or patient CSF-mediated induction of mHTT-GFP aggregation could be modified by elevated DNAJB6 expression ${ }^{43}$. We found that DNAJB6 overexpression almost completely abolished the recombinant mHTT seed-induced aggregation (Fig. 6a). Similarly, we observed a significant DNAJB6-mediated suppression of mHTT-GFP aggregation induced by the HD patient CSF (Fig. 6b). However, no difference is observed in the groups treated with CSF from AD and PD patients with or without DNAJB6 overexpression (Fig. 6b). The results not only further confirmed that $\mathrm{AD}$ and PD CSF only induce neglectable mHTT seeding activities, if any, in our cellular assay, but also showed that such baseline seeding activities are not template-dependent and thus not sensitive to elevated DNAJB6 levels. Together, our findings suggest that induced aggregation by HD CSF is likely a DNAJB6-sensitive, mHTT seed-mediated nucleation and fibrillation process.

The utilization of epitope-specific antibodies to block protein-protein interaction or to deplete mutant proteins from the biosamples is a well-established strategy to evaluate the molecular nature of pathological seeding 
a

\begin{tabular}{|c|c|c|c|}
\hline \multicolumn{2}{|c|}{$\mathrm{N}$-termQ } & \multirow{2}{*}{$\begin{array}{l}\text { EM48 } \\
\text { MAB5492 } \\
\text { MW7 } \\
\text { polyP }\end{array}$} & \\
\hline $\begin{array}{l}-19 a a \\
\text { N17 }\end{array}$ & \begin{tabular}{|l|}
$\mathrm{C} 2$ \\
$3 \mathrm{~B} 5 \mathrm{H} 10$
\end{tabular} & & Ex1 C-term \\
\hline N17 & polyQ & polyP & \\
\hline
\end{tabular}

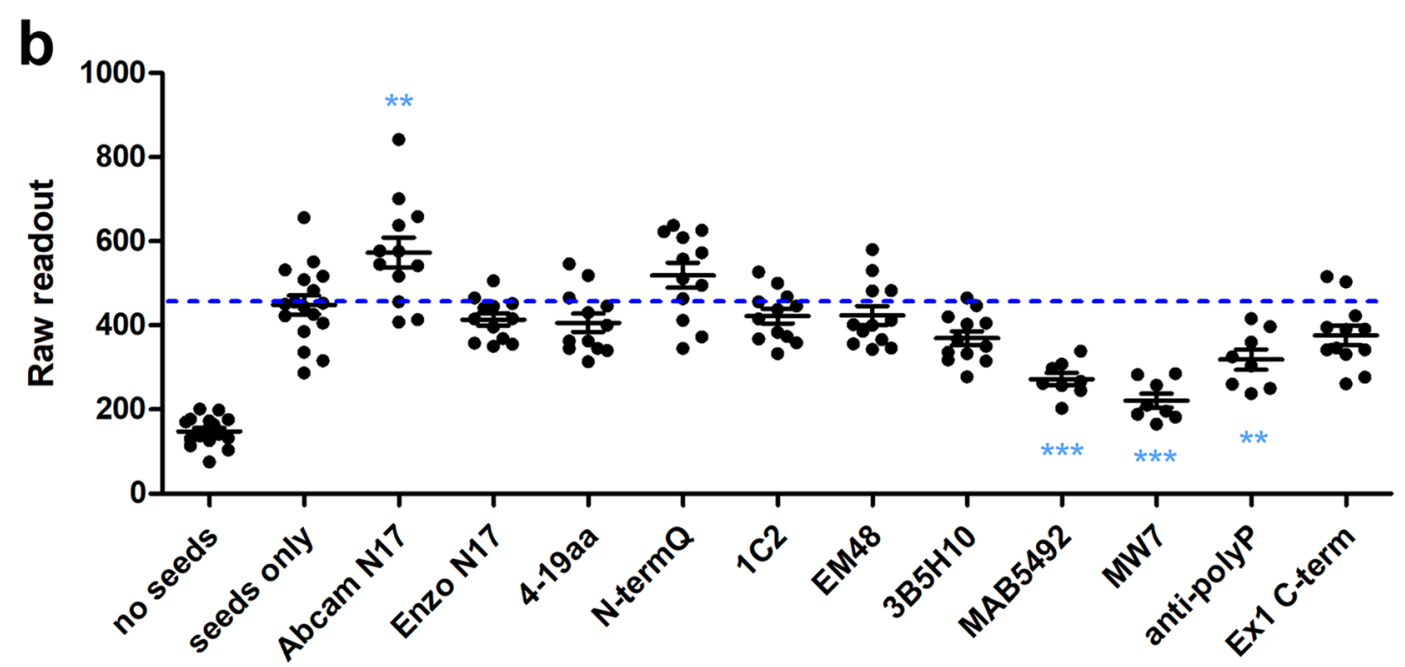

C

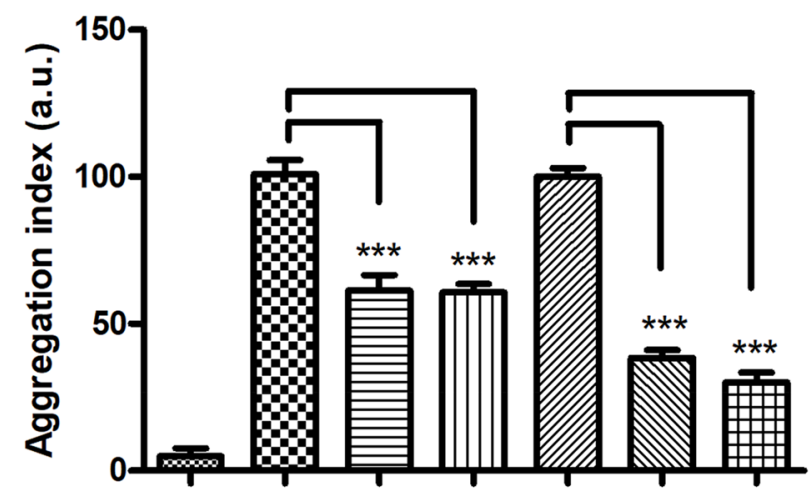

$\begin{array}{llllllll}\text { HD CSF } & - & + & + & + & - & - & - \\ \text { 51Q seeds } & - & - & - & - & + & + & + \\ \text { MW7 } & - & - & + & - & - & + & - \\ \text { MAB5492 } & - & - & - & + & - & - & +\end{array}$

Figure 7. Only anti-polyP antibodies were capable of blocking the seeding activities of HD patient CSF. (a) An illustration indicates the epitopes for the HTT antibodies. (b) The mHTT-51Q seeds were preincubated with the indicated antibodies before administration to mHTT- $\triangle$ N17-GFP cells in the 384-well format. The epitopes of the antibodies are indicated in the upper panel. Confocal images were acquired with automation 5 days after seeding and batch analyzed. Statistic comparisons were made between the antibody-treated groups with the mHTT-51Q seed-only group. $n=8-12$. (c) The mHTT-51Q seeds and postmortem CSF from HD patients were immunodepleted with two anti-polyP antibodies (as indicated) overnight and applied to mHTT- $\Delta$ N17-GFP cells for 5 days. $n=6$. Statistic results are presented as mean \pm s.e.m. ${ }^{* \star} p<0.01 ;{ }^{\star \star \star} p<0.001$.

activities, and has been shown to effectively inhibit the intracellular aggregation and propagation of the disease proteins, e.g. aSyn and Tau, by extracellularly administered antibodies ${ }^{44,45}$. To investigate which mHTT-exon 1 domains may be critical in mediating the template-based seeding activities described above, we tested a battery of antibodies against different domains of mHTT-exon 1 (Fig. 7a). Three out of four antibodies against the polyproline-rich domain (polyP) significantly reduced mHTT-GFP aggregation induced by the recombinant mHTT seeds (Fig. 7b). In contrast, none of antibodies against the N17 domain or the polyQ domain could block the mHTT seeding activity (Fig. 7b). In fact, one of the three anti-N17 antibodies (i.e. Abcam anti-N17) even slightly but significantly enhanced the nucleation. Although the blocking efficiency may vary among antibodies 
against same epitopes, the findings suggest that the polyP domain is critical for recombinant mHTT seeds to elicit template-based aggregation of mHTT- $\triangle \mathrm{N} 17-\mathrm{GFP}$ intracellularly.

We next tested whether the CSF mHTT seeding species could be immunodepleted by anti-polyP antibodies to reduce their seeding propensity. Two anti-polyP antibodies (MW7 and MAB5492) were used to immunodeplete mHTT seeding species from the HD CSF samples. The immunodepleted CSF samples were then applied to the mHTT- $\triangle$ N17-GFP cells. We found that HD patient CSF, immunodepleted by both anti-polyP antibodies, significantly diminished its seeding ability in the assay (Fig. 7c). This result strongly supports that the seeding activities presenting in the HD patient CSF samples are consisted of HTT species with an exposed polyP domain, and this domain is necessary to mediate the seed-induced aggregation in the reporter cells.

\section{Discussion}

To specifically detect mHTT seeding species, especially those in HD patient CSF, we developed a sensitive reporter cell assay with high-throughput capability. Two key genetic engineering of the reporter protein are the use of moderate CAG repeats (46Q) for minimal aggregation at the baseline and deletion of the N17 domain, which showed remarkable enhancement in sensitivity for detecting mHTT seeding species, including those in HD patient biosamples. Furthermore, by adapting the new cell line into a 384-well, high-content imaging assay platform, we are able to reproducibly detect and quantify mHTT seeding activities in the HD patient CSF. Our preliminary study of clinical samples reveals a significant increase in the mHTT seeding activities in the CSF of HD gene carriers upon their initial diagnosis of HD (i.e. DCL4), and a continuing increase of such seeding activities with progression of neuropathological grades in the postmortem samples. Finally, our assay reveals the mHTT seeding activities could be inhibited by overexpression of the chaperone DNAJB6 and by immunodepletion using antibodies against the polyP domain of mHTT. Further studies of larger premanifest and manifest HD patient cohort are needed to fully evaluate the utility of longitudinal CSF mHTT seeding activities, as detected by the highly sensitive cell-based assay, in tracking the clinical onset and progression of HD. Finally, our study also demonstrated the utility of this cell-based assay to evaluate molecules that can suppress the mHTT seeding activities from HD patient CSF, which in turn could be further investigated for their roles in disease modifications.

One important finding of this study is that the propensity of the mHTT seeding is relatively minimal in HD gene carriers prior to the symptom onset, and is significantly elevated upon disease diagnosis and appears to continuously increase with neuropathological progression. Previous studies have reported increased mHTT species in the CSF before and after the disease onset ${ }^{9,10,26,46}$. However, changes in the levels of mHTT seeding species have not been described over the course of the disease and with the concurrence of symptoms. In this study, we demonstrate the neuropathological and clinical stage-dependent increase (i.e. with HD grades in postmortem CSFs and with DCL scores in the PREDIT-HD cohort, respectively) in mHTT CSF seeding activities. Despite the limited availability of multi-visit samples in PREDICT-HD, our study also showed that the seeding propensity of CSF is also augmented in subsequent visits of HD patients but not in those of premanifest mutant carriers. Moreover, our preliminary findings showed the CSF seeding propensity is positively correlated with the disease severity, including TFC and total motor score, similar to the currently clinically used SMC assay ${ }^{10,11}$. The SMC assay is significantly correlated with the CAP score ${ }^{10,41}$. One important question is whether the CSF mHTT seeding activities are tightly correlated with the CAG length and/or age of the patients. We found that the CSF mHTT seeding propensity does not appear to correlate with the CAP score of the patients. Thus, our assay does not seem to probe the same mHTT species in the patient CSF as the SMC assay. We acknowledge that a limitation of our study is the relatively small number of CSF samples from premanifest and manifest HD patients used. Therefore, our findings related to the relationships of CSF seeding activities and clinical disease stages should be considered preliminary. Future large-scale studies with longitudinal CSF samples and clinical data from both HD gene carriers and patients is needed to rigorously evaluate the clinical utility of this high-throughput, cell-based mHTT seeding assay in monitoring HD onset and progression and in evaluating efficacies of HD therapeutics.

We demonstrated that overexpression of DNAJB6 in the reporter cell line can block the seeding activities from preformed seeds and patient CSF. These findings confirmed that our assay is detecting template-based, aggregate-inducing activities of mHTT species in the CSF. It further suggests that studying chaperone-mediated mHTT proteostasis, such as those mediated by DNAJB6 and DNAJB8 ${ }^{42,43}$ and other chaperones ${ }^{47}$, could be a meaningful approach to identify molecular targets and candidate therapeutic reagents to block mHTT seeding in the patients.

A key advance in our study is to provide strong evidence that the CSF seeding activities from HD patients are likely to be mediated by specific mHTT species, of which the polyP region is exposed and might play a key role in the seeding process. We showed that antibody-blockade and immunodepletion with three different anti-polyP antibodies could significantly reduce mHTT-GFP aggregation induced by recombinant seed and CSF from HD patients, while anti-N17 or anti-polyQ antibodies could not elicit similar effects. This result is consistent with prior studies showing that co-expressing polyP-specific intrabodies inhibited aggregation of mHT proteins in HD transgenic mice ${ }^{4-50}$. It is worth noting that a commonly used anti-HTT antibody, EM48, of which epitope is mapped to the polyP region, could not elicit the same blocking effect. This might be attributed to the intrinsic difference of antibodies in epitope recognition and affinity. Recent structure studies suggest a "bottle brush" model for HTT exon 1 structure, with the polyP domain forms bristled helix-rich structures projecting away from the fibril core of N17 and polyQ ${ }^{51,52}$. Aggregation of mHTT is a stepwise event, initiated by N17 interaction, followed by $\beta$-sheet formation of polyQ and, lastly, crowding of the polyP bristles. Given the slowest step lies on the final structure maturation as polyP crowding ${ }^{52}$, presence of antibodies against the polyP domain might reduce $\mathrm{mHTT}$-GFP aggregation through interference with polyP bristles compaction. Future studies are needed to test whether extracellular delivery of anti-HTT-polyP antibodies could be an effective measure to block the propagation of mHTT aggregation and HD pathogenesis in vivo. 
Finally, our scalable, highly sensitive cell-based mHTT seeding assay can be used in large scale perturbation studies, e.g. anti-HTT antibodies, intracellular gene-based screenings (i.e. shRNA, CRISPR/Cas9, or cDNA overexpression) and small molecule screenings to systematically identify molecules that could modulate mHTT seeding activities. A good example is the antibody screening reported in this paper. This is made possible as our assay requires only very small amount of CSF (i.e. 10-20 $\mu$ l) per sample. Thus, future large-scale, phenotypedriven screening with our assay could identify genetic targets or small molecules to curb such pathogenic activities in the patients.

In conclusion, we reported the development of a novel high-throughput cell-based system that could sensitively and specifically detect disease-related mHTT seeding activities in HD patient CSF. By using this platform, we identified molecules (i.e. DNAJB6 expression or anti-HTT polyP antibodies) that could block such seeding activities. Our study will enable future systematic analyses of the pathogenic mHTT seeding activities in HD patient CSF to evaluate its potential utility as disease-progression biomarkers, and for large-scale screening of molecules to ameliorate pathogenic seeding activities in HD.

\section{Methods}

Human brain and CSF samples. Prior to the seeding assays, samples were randomized and blinded to investigators. Autopsy brain tissues of subjects with HD were obtained from UCLA Human Brain and Spinal Fluid Resource Center (Supplemental Table S1). Postmortem CSF was provided by the New York Brain Bank at Columbia University. Vonsattel neuropathological grades of the HD patients was determined by the New York Brain Bank using their respective brain tissues (Supplemental Table S2). PREDICT CSF samples were obtained from BioSEND at Indiana University. Correlated clinical information and scores were provided by Dr. Jane Paulsen at University of Iowa via dbGaP (Supplemental Table S3) ${ }^{40}$. The PREDICT-HD participants visits the centers annually for biosample collection (e.g. blood, CSF and saliva), brain imaging and cognitive and other neurological evaluation ${ }^{53,54}$. CSF were collected through lumbar puncture (LP) after fasting. Basic CSF quality analyses were conducted for cell count, erythrocytes, total protein, and glucose ${ }^{54}$. All paired multi-visit CSF samples from the same individuals were collected 1 year apart.

Animals. Heterozygous Q175 mHtt knock-in mice carrying mHtt with about $190 \mathrm{CAG}$ repeats were obtain from Jackson Laboratory and aged to designated ages. Animals were housed in standard mouse cages under conventional laboratory conditions, with constant temperature and humidity, $12 \mathrm{~h} / 12 \mathrm{~h}$ light/dark cycle and food and water ad libitum. All animal studies were carried out in strict accordance with National Institutes of Health guidelines and approved by the UCLA Institutional Animal Care and Use Committees. The mice were anesthetized by pentobarbital. Brains were removed and carefully dissected to obtain cortical and striatal tissues. Dissected tissues were snap frozen in dry ice and stored in $-80^{\circ} \mathrm{C}$ before further processing.

Antibodies. Anti-HTT antibodies EM48, 1C2, 3B5H10 and MAB5492 were obtained from Millipore. An anti-HTT antibody MW7 were from the Developmental Studies Hybridoma Bank. Two anti-N17 antibody were purchased from Abcam and Enzo. Anti-N-termQ, anti-4-19aa, anti-polyP and anti-Ex1 C-term of mHTT antibodies were provided by CHDI foundation.

Brain lysate preparation. Cortical and striatal samples of HD patients or mice were submerged in PBS and homogenized using a Dounce homogenizer. Homogenates were then sonicated for two $10 \mathrm{~s}$-on/10 s-off cycles and centrifuged at $3000 \times g$ for $10 \mathrm{~min}$ to remove undissolved tissue debris. Supernatants were re-sonicated and stored at $-80^{\circ} \mathrm{C}$ until use.

Generation of cell lines and cell culture. DNA constructs of mHTT exon 1 (46Q) fragment C-terminally tagged with EGFP were cloned into a mammalian expression vector, pcDNA3.1, to generate the mHTT46Q-GFP plasmid. mHTT-SD-GFP and $\mathrm{mHTT}-\Delta \mathrm{N} 17-\mathrm{GFP}$ plasmids were generated by direct point mutation of S13/S16 residues to D13/D16 and deletion of 2-16 a.a. residues of mHTT on the mHTT-46Q-GFP plasmid using a QuikChange site-directed mutagenesis kit (Agilent), respectively. Resulting constructs were validated by Sanger's sequencing. For generation of stable reporter cell lines, HEK293 cells (ATCC CRL-1573, Manassas, VA) were transfected with one of aforementioned plasmids using FuGENE HD (Promega) as described by the manufacturer's protocol. Two days following transfection, the culture media was replenished and G418 (500 ng/ $\mathrm{ml}$ ) was administered, allowing the selective propagation of transfected cells in culture. Neomycin resistant, fluorescent-labeled colonies were identified by a fluorescent microscope on day 14. Single transgenic colonies were picked by a micropipette, and transferred into a 24 -well culture dish. The cells continuously proliferated in the presence of G418 (reduced to $200 \mathrm{ng} / \mathrm{ml}$ ), and the expression of transgenes was monitored by a fluorescent microscope. Cellular expression of mHTT-GFP was confirmed by immunostaining using an anti-HTT antibody, EM48 (Millipore). Stable cells were maintained in Dulbecco's modified Eagle's medium (DMEM; ThermoFisher) supplemented with $10 \%$ fetal bovine serum (FBS) and gentamycin $(25 \mathrm{ng} / \mathrm{ml})$ at $37^{\circ} \mathrm{C}$.

Generation of recombinant proteins and seeds. Recombinant mHTT-51Q proteins were produced as previously described ${ }^{30}$. In brief, Rosetta 2 (DE3) pLysS competent cells expressing pGEX-mHtt-Ex1-Q51 plasmid were induced with $1 \mathrm{mM}$ IPTG for $2.5 \mathrm{~h}$ at $16^{\circ} \mathrm{C}$. Cell pellets were lysed using an Emulsiflex (Avestin, Ottowa, Canada) and incubated with GSH-Sepharose resin (GE Healthcare, Pittsburgh, PA, USA) and washed with $0.1 \%$ Triton, $500 \mathrm{mM} \mathrm{NaCl}$, and $5 \mathrm{mM} \mathrm{Mg}$-ATP before eluting protein with $15 \mathrm{mM}$ Glutathione. Protein was concentrated and buffer exchanged with $50 \mathrm{mM}$ Tris- $\mathrm{HCl}, \mathrm{pH} 8.0 ; 100 \mathrm{mM} \mathrm{NaCl} ; 5 \%$ glycerol. Con- 
centrated mHTT-51Q protein was $0.2 \mu \mathrm{m}$ PVDF filtered. Aggregation reaction was conducted by incubating $20 \mu \mathrm{M}$ of mHTT-51Q in TEV reaction buffer (ThermoFisher) at $30^{\circ} \mathrm{C}$ for $60 \mathrm{~h}$. Aggregates were centrifuged at $16,000 \times g$ for $1 \mathrm{~h}$ at room temperature to pellet fibers, which were resuspended in TEV buffer and then sonicated $1 \mathrm{~s}$-on/1 s-off pulsing using a Fisher Scientific 120W Sonic Dismembrator (ThermoFisher). The size of the sonicated fibers was measured by DLS using a Zetasizer Nano ZS (Malvern, Worcestershire, UK).

$\mathrm{A} \beta$ peptides corresponding to the human $\mathrm{A} \beta$ amino acids 1-42 were purchased from American Peptide Co. (Sunnyvale, CA) and dissolved to a final concentration of $1 \mathrm{mg} / \mathrm{ml}$ in DMSO. To generate $\mathrm{fA} \beta$ seeds, lyophilized $\mathrm{A} \beta$ peptides were reconstituted in sterile distilled water and incubated at $37^{\circ} \mathrm{C}$ for 1 week to allow fibrillization. $\mathrm{fA} \beta$ was then sonicated for two $10 \mathrm{~s}$-on/10 s-off cycles to generate fA $\beta$ seeds.

Cell-based seeding assay. One day prior to seeding, cells were plated on poly-D-lysine coated coverslips or 384-well plates and cultured with DMEM plus 10\% FBS. mHTT-51Q $(2 \mu \mathrm{g} / \mathrm{ml}$ or designated concentrations), $\mathrm{A} \beta(10 \mu \mathrm{g} / \mathrm{ml})$, Tau $(10 \mu \mathrm{g} / \mathrm{ml})$, brain extract $(10 \mu \mathrm{g} / \mathrm{ml}$ of total protein) or CSF (at final 1:20 or other designated dilutions) were directly applied to the cells. For DNAJB6 experiments, cells were transfected with a DNAJB6 expressing plasmid pcDNA5/FRT/TO DNAJB66 ${ }^{43}$ a day prior to seeding. The media were then replenished and recombinant $\mathrm{mHTT}$ seeds $(2 \mu \mathrm{g} / \mathrm{ml})$ or CSF (1:20; UCLA in-house collection) were added. For evaluating the role of the mHTT domains in seed-induced aggregation, two methods were used to apply mHTT domainspecific antibodies: (1) immunoblocking - the recombinant seeds were preincubated with antibodies $(2 \mu \mathrm{g} / \mathrm{ml})$ for 10 min before applying to the cells; (2) immunodepletion-recombinant mHTT seeds or CSF (UCLA's neuropathological collection) were incubated with $2 \mu \mathrm{g}$ of antibodies and $10 \mu \mathrm{l}$ of Protein A/G agarose beads in $100 \mu \mathrm{l}$ of PBS overnight. The mixture were then centrifuged at $13,000 \times g$ for $2 \mathrm{~min}$. The resulting supernatant were applied to the cells at the concentration as if they had not been immunodepleted (seeds at $2 \mu \mathrm{g} / \mathrm{ml}$ and CSF at 1:20). The cells were then incubated for 3 or 5 days to allow aggregate formation for low throughput (i.e. coverslips) or high throughput (i.e. 384-wells) experiments, respectively. Confocal images were taken using a Zeiss LSM 500 confocal or ImageXpress Micro Confocal (Molecular Devices) and analyzed by ImageJ or MetaXpress (Molecular Devices), respectively. The particle measurement function in ImageJ were used to quantify the aggregates in the cells. High-content image analysis is described below.

High-content imaging and analysis. High-content images were acquired using automated the ImageXpress Micro Confocal system with a $10 \times$ objective at the confocal slit mode. Z-stacked images were taken to ensure capturing all aggregates within the cells. Four sites per well were imaged to avoid in-well variation. Maximum intensity projected images were analyzed by MetaXpress with Granularity module at Standard analysis mode. Raw readouts were normalized to the internal controls within each plate. The normalized values are labeled as "aggregation index" in the figures. For all experiments, 6-8 wells were used as the technical replica and averaged to generate one data point for a biological replicate.

Immunodepletion of mHTT seeding species from CSF. HD patient CSF (1:10) were mixed with $2 \mu \mathrm{g}$ of an anti-HTT antibody and Protein A/G-conjugated agarose beads (1:10, ThermoFisher) in DMEM and incubated with rocking for $24 \mathrm{~h}$ at $4{ }^{\circ} \mathrm{C}$. The mixture were centrifuged at $13,000 \times g$ for $2 \mathrm{~min}$. The supernatants were then immediately applied to the cells and incubated for designated time for the seeding assay.

Statistical analyses. Group comparisons without covariates were performed using one-way ANOVA with Tukey post-hoc test. To assess possible confounders, linear models were used for post-mortem CSF data, while linear mixed effect models were used for PREDICT-HD data to account for the fact that there are two data points (visits 1 year apart) for each subject. Bonferroni correction was used to account for testing of 82 clinical scores. Shapiro-Wilks test was used to test for normality of residuals. Pearson correlation and the corresponding significance estimate were used to quantify association between continuous variables.

Received: 30 June 2020; Accepted: 28 October 2020

Published online: 20 November 2020

\section{References}

1. DiFiglia, M. et al. Aggregation of huntingtin in neuronal intranuclear inclusions and dystrophic neurites in brain. Science 277, 1990-1993. https://doi.org/10.1126/science.277.5334.1990 (1997).

2. Arrasate, M., Mitra, S., Schweitzer, E. S., Segal, M. R. \& Finkbeiner, S. Inclusion body formation reduces levels of mutant huntingtin and the risk of neuronal death. Nature 431, 805-810. https://doi.org/10.1038/nature02998 (2004).

3. Gu, X. et al. Pathological cell-cell interactions are necessary for striatal pathogenesis in a conditional mouse model of Huntington's disease. Mol. Neurodegener. 2, 8. https://doi.org/10.1186/1750-1326-2-8 (2007).

4. Gu, X. et al. Pathological cell-cell interactions elicited by a neuropathogenic form of mutant huntingtin contribute to cortical pathogenesis in HD mice. Neuron 46, 433-444. https://doi.org/10.1016/j.neuron.2005.03.025 (2005).

5. Bauerlein, F. J. B. et al. In situ architecture and cellular interactions of PolyQ inclusions. Cell 171, 179-187. https://doi.org/10.1016/j. cell.2017.08.009 (2017).

6. Pircs, K. et al. Huntingtin aggregation impairs autophagy, leading to Argonaute-2 accumulation and global MicroRNA dysregulation. Cell Rep. 24, 1397-1406. https://doi.org/10.1016/j.celrep.2018.07.017 (2018).

7. Ramdzan, Y. M. et al. Huntingtin inclusions trigger cellular quiescence, deactivate apoptosis, and lead to delayed necrosis. Cell Rep. 19, 919-927. https://doi.org/10.1016/j.celrep.2017.04.029 (2017).

8. Li, H., Li, S. H., Johnston, H., Shelbourne, P. F. \& Li, X. J. Amino-terminal fragments of mutant huntingtin show selective accumulation in striatal neurons and synaptic toxicity. Nat. Genet. 25, 385-389. https://doi.org/10.1038/78054 (2000). 
9. Byrne, L. M. et al. Evaluation of mutant huntingtin and neurofilament proteins as potential markers in Huntington's disease. Transl. Med. Sci. https://doi.org/10.1126/scitranslmed.aat7108 (2018).

10. Wild, E. J. et al. Quantification of mutant huntingtin protein in cerebrospinal fluid from Huntington's disease patients. J. Clin. Invest. 125, 1979-1986. https://doi.org/10.1172/JCI80743 (2015).

11. Tabrizi, S. J. et al. Targeting huntingtin expression in patients with Huntington's disease. N. Engl. J. Med. 380, 2307-2316. https:// doi.org/10.1056/NEJMoa1900907 (2019).

12. Macdonald, D. et al. Quantification assays for total and polyglutamine-expanded huntingtin proteins. PLoS ONE 9, e96854. https ://doi.org/10.1371/journal.pone.0096854 (2014).

13. Reindl, W. et al. Meso scale discovery-based assays for the detection of aggregated huntingtin. PLoS ONE 14, e0213521. https:// doi.org/10.1371/journal.pone.0213521 (2019).

14. Hensman Moss, D. J. et al. Quantification of huntingtin protein species in Huntington's disease patient leukocytes using optimised electrochemiluminescence immunoassays. PLoS ONE 12, e0189891. https://doi.org/10.1371/journal.pone.0189891 (2017).

15. Southwell, A. L. et al. Ultrasensitive measurement of huntingtin protein in cerebrospinal fluid demonstrates increase with Huntington disease stage and decrease following brain huntingtin suppression. Sci. Rep. 5, 12166. https://doi.org/10.1038/srep12166 (2015).

16. Gray, M. et al. Full-length human mutant huntingtin with a stable polyglutamine repeat can elicit progressive and selective neuropathogenesis in BACHD mice. J. Neurosci. 28, 6182-6195. https://doi.org/10.1523/JNEUROSCI.0857-08.2008 (2008).

17. Lee, C. Y., Cantle, J. P. \& Yang, X. W. Genetic manipulations of mutant huntingtin in mice: new insights into Huntington.s disease pathogenesis. FEBS J. 280, 4382-4394. https://doi.org/10.1111/febs.12418 (2013).

18. Ratovitski, T. et al. Post-translational modifications (PTMs), identified on endogenous huntingtin, cluster within proteolytic domains between HEAT repeats. J. Proteome Res. 16, 2692-2708. https://doi.org/10.1021/acs.jproteome.6b00991 (2017).

19. Pearce, M. M. P., Spartz, E. J., Hong, W., Luo, L. \& Kopito, R. R. Prion-like transmission of neuronal huntingtin aggregates to phagocytic glia in the Drosophila brain. Nat. Commun. 6, 6768. https://doi.org/10.1038/ncomms7768 (2015).

20. Polymenidou, M. \& Cleveland, D. W. Prion-like spread of protein aggregates in neurodegeneration. J. Exp. Med. 209, 889-893. https://doi.org/10.1084/jem.20120741 (2012).

21. Thakur, A. K. et al. Polyglutamine disruption of the huntingtin exon $1 \mathrm{~N}$ terminus triggers a complex aggregation mechanism. Nat. Struct. Mol. Biol. 16, 380-389. https://doi.org/10.1038/nsmb.1570 (2009).

22. Ren, P. H. et al. Cytoplasmic penetration and persistent infection of mammalian cells by polyglutamine aggregates. Nat. Cell Biol. 11, 219-225. https://doi.org/10.1038/ncb1830 (2009).

23. Yang, W., Dunlap, J. R., Andrews, R. B. \& Wetzel, R. Aggregated polyglutamine peptides delivered to nuclei are toxic to mammalian cells. Hum. Mol. Genet. 11, 2905-2917. https://doi.org/10.1093/hmg/11.23.2905 (2002).

24. Ast, A. et al. mHTT seeding activity: a marker of disease progression and neurotoxicity in models of Huntington's disease. Mol. Cell 71, 675-688. https://doi.org/10.1016/j.molcel.2018.07.032 (2018).

25. Morozova, O. A., Gupta, S. \& Colby, D. W. Prefibrillar huntingtin oligomers isolated from HD brain potently seed amyloid formation. FEBS Lett. 589, 1897-1903. https://doi.org/10.1016/j.febslet.2015.05.041 (2015).

26. Tan, Z. et al. Huntington's disease cerebrospinal fluid seeds aggregation of mutant huntingtin. Mol. Psychiatry 20, 1286-1293. https ://doi.org/10.1038/mp.2015.81 (2015).

27. Keum, J. W. et al. The HTT CAG-expansion mutation determines age at death but not disease duration in huntington disease. Am. J. Hum. Genet. 98, 287-298. https://doi.org/10.1016/j.ajhg.2015.12.018 (2016).

28. Ramdzan, Y. M. et al. Conformation sensors that distinguish monomeric proteins from oligomers in live cells. Chem. Biol. 17, 371-379. https://doi.org/10.1016/j.chembiol.2010.03.011 (2010).

29. Gu, X. et al. N17 Modifies mutant Huntingtin nuclear pathogenesis and severity of disease in HD BAC transgenic mice. Neuron 85, 726-741. https://doi.org/10.1016/j.neuron.2015.01.008 (2015).

30. Shen, K. et al. Control of the structural landscape and neuronal proteotoxicity of mutant Huntingtin by domains flanking the polyQ tract. eLife https://doi.org/10.7554/eLife.18065 (2016).

31. Crick, S. L., Ruff, K. M., Garai, K., Frieden, C. \& Pappu, R. V. Unmasking the roles of N- and C-terminal flanking sequences from exon 1 of huntingtin as modulators of polyglutamine aggregation. Proc. Natl. Acad. Sci. U. S. A. 110, 20075-20080. https://doi. org/10.1073/pnas.1320626110 (2013).

32. Gu, X. et al. Serines 13 and 16 are critical determinants of full-length human mutant huntingtin induced disease pathogenesis in HD mice. Neuron 64, 828-840. https://doi.org/10.1016/j.neuron.2009.11.020 (2009).

33. Mishra, R. et al. Serine phosphorylation suppresses huntingtin amyloid accumulation by altering protein aggregation properties. J. Mol. Biol. 424, 1-14. https://doi.org/10.1016/j.jmb.2012.09.011 (2012).

34. Veldman, M. B. et al. The N17 domain mitigates nuclear toxicity in a novel zebrafish Huntington's disease model. Mol. Neurodegener 10, 67. https://doi.org/10.1186/s13024-015-0063-2 (2015)

35. Heikkinen, T. et al. Characterization of neurophysiological and behavioral changes, MRI brain volumetry and 1H MRS in zQ175 knock-in mouse model of Huntington's disease. PLoS ONE 7, e50717. https://doi.org/10.1371/journal.pone.0050717 (2012).

36. Menalled, L. B. et al. Comprehensive behavioral and molecular characterization of a new knock-in mouse model of Huntington's disease: zQ175. PLoS ONE 7, e49838. https://doi.org/10.1371/journal.pone.0049838 (2012).

37. Smith, G. A. et al. Progressive axonal transport and synaptic protein changes correlate with behavioral and neuropathological abnormalities in the heterozygous Q175 KI mouse model of Huntington's disease. Hum. Mol. Genet. 23, 4510-4527. https://doi. org $/ 10.1093 / \mathrm{hmg} / \mathrm{ddu} 166(2014)$.

38. Langfelder, P. et al. Integrated genomics and proteomics define huntingtin CAG length-dependent networks in mice. Nat. Neurosci. 19, 623-633. https://doi.org/10.1038/nn.4256 (2016).

39. Vonsattel, J. P. et al. Neuropathological classification of Huntington's disease. J. Neuropathol. Exp. Neurol. 44, 559-577. https://doi. org/10.1097/00005072-198511000-00003 (1985).

40. Long, J. D., Paulsen, J. S., Investigators, P.-H. \& Coordinators of the Huntington Study, G. Multivariate prediction of motor diagnosis in Huntington's disease: 12 years of PREDICT-HD. Mov. Disord. 30, 1664-1672. https://doi.org/10.1002/mds.26364 (2015).

41. Ross, C. A. et al. Huntington disease: natural history, biomarkers and prospects for therapeutics. Nat. Rev. Neurol. 10, $204-216$. https://doi.org/10.1038/nrneurol.2014.24 (2014).

42. Gillis, J. et al. The DNAJB6 and DNAJB8 protein chaperones prevent intracellular aggregation of polyglutamine peptides. J. Biol. Chem. 288, 17225-17237. https://doi.org/10.1074/jbc.M112.421685 (2013).

43. Kakkar, V. et al. The S/T-Rich Motif in the DNAJB6 chaperone delays polyglutamine aggregation and the onset of disease in a mouse model. Mol. Cell 62, 272-283. https://doi.org/10.1016/j.molcel.2016.03.017 (2016).

44. Yanamandra, K. et al. Anti-tau antibodies that block tau aggregate seeding in vitro markedly decrease pathology and improve cognition in vivo. Neuron 80, 402-414. https://doi.org/10.1016/j.neuron.2013.07.046 (2013).

45. Masliah, E. et al. Effects of alpha-synuclein immunization in a mouse model of Parkinson’s disease. Neuron 46, 857-868. https:// doi.org/10.1016/j.neuron.2005.05.010 (2005).

46. Fodale, V. et al. Validation of ultrasensitive mutant huntingtin detection in human cerebrospinal fluid by single molecule counting immunoassay. J. Huntingtons Dis. 6, 349-361. https://doi.org/10.3233/JHD-170269 (2017).

47. Tam, S. et al. The chaperonin TRiC blocks a huntingtin sequence element that promotes the conformational switch to aggregation. Nat. Struct. Mol. Biol. 16, 1279-1285. https://doi.org/10.1038/nsmb.1700 (2009). 
48. Khoshnan, A., Ko, J. \& Patterson, P. H. Effects of intracellular expression of anti-huntingtin antibodies of various specificities on mutant huntingtin aggregation and toxicity. Proc. Natl. Acad. Sci. U. S. A. 99, 1002-1007. https://doi.org/10.1073/pnas.022631799 (2002).

49. Ko, J. et al. Identification of distinct conformations associated with monomers and fibril assemblies of mutant huntingtin. Hum. Mol. Genet. 27, 2330-2343. https://doi.org/10.1093/hmg/ddy141 (2018).

50. Southwell, A. L. et al. Intrabodies binding the proline-rich domains of mutant huntingtin increase its turnover and reduce neurotoxicity. J. Neurosci. 28, 9013-9020. https://doi.org/10.1523/JNEUROSCI.2747-08.2008 (2008).

51. Isas, J. M., Langen, R. \& Siemer, A. B. Solid-state nuclear magnetic resonance on the static and dynamic domains of huntingtin exon-1 fibrils. Biochemistry 54, 3942-3949. https://doi.org/10.1021/acs.biochem.5b00281 (2015).

52. Pandey, N. K. et al. The 17-residue-long $\mathrm{N}$ terminus in huntingtin controls stepwise aggregation in solution and on membranes via different mechanisms. J. Biol. Chem. 293, 2597-2605. https://doi.org/10.1074/jbc.M117.813667 (2018).

53. Paulsen, J. S. et al. Preparing for preventive clinical trials: the Predict-HD study. Arch. Neurol. 63, 883-890. https://doi.org/10.1001/ archneur.63.6.883 (2006)

54. Reed, E. R. et al. MicroRNAs in CSF as prodromal biomarkers for Huntington disease in the PREDICT-HD study. Neurology $\mathbf{9 0}$, e264-e272. https://doi.org/10.1212/WNL.0000000000004844 (2018).

\section{Acknowledgements}

We would like to express our highest appreciation to all the participants from the HD and related disease community for generous donation of CSF samples and postmortem samples used in this research. The research was supported by NINDS/NIH (NS074312), the Leslie Gehry Brenner Award from Hereditary Disease Foundation, a CTSI voucher award for UCLA MSSR core, and an anonymous donation from an HD family. X.W.Y. is supported by the Terry Semel Chair in Alzheimer's Disease Research and Treatment at UCLA. The Yang Lab is also supported by NIH Grants (NS074312, NS084298, MH106008, AG056114), David Weill Fund from the Jane and Terry Semel Institute at UCLA, the Hereditary Disease Foundation, and the CHDI Foundation, Inc. We thank the help from Drs. Tatiana Foroud and Scott Kaiser at BioSEND and Drs. Christine Swanson-Fischer and Daniel Miller at NINDS for coordinating the acquirement of the PREDICT-HD samples. We would like to thank Dr. Jane Paulsen at University of Iowa for sharing the PREDICT-HD clinical dataset. We thank Dr. Ake Lu for the advise of analyzing patient CSF-related data. We acknowledge the help from the NINDS Informatics Center Grant (P30 NS062691), and UCLA MSSR Core.

\section{Author contributions}

X.W.Y. provided the conceptual framework and provide funds for the study. C.Y.D.L. and X.W.Y. designed the experiments and interpreted the results. C.Y.D.L. and X.W.Y. wrote the manuscript. C.Y.D.L. generated the cell lines. C.Y.D.L., N.W. and R.D. established the assay. K.S. and J.F. contributed to the generation of recombinant mHTT species and provide consultation to the seeding assay. C.Y.D.L. performed experiments and analyzed data shown in all figures. N. Wang contributed to Figs. 1c and 2. M.S. performed experiments and analyzed data shown in Fig. 1d,e, 3a and Supplemental Fig. S1. P.L. performed analyses for Fig. 5e, Supplemental Fig. S3 and Tables S4-S8. K.H.C. contributed to Fig. 6. H.V.V. collected and performed clinical analysis of the autopsy samples from UCLA. E.P.C. and J.P.V. performed neuropathological staging of the autopsy samples and provided corresponding postmortem CSF samples from New York Brain Bank at Columbia University. N.S.W. contributed to the design of the experiment using the postmortem CSF samples from neuropathologically-graded HD cases.

\section{Competing interests}

The authors declare no competing interests.

\section{Additional information}

Supplementary information is available for this paper at https://doi.org/10.1038/s41598-020-77164-1.

Correspondence and requests for materials should be addressed to X.W.Y.

Reprints and permissions information is available at www.nature.com/reprints.

Publisher's note Springer Nature remains neutral with regard to jurisdictional claims in published maps and institutional affiliations.

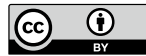

Open Access This article is licensed under a Creative Commons Attribution 4.0 International License, which permits use, sharing, adaptation, distribution and reproduction in any medium or format, as long as you give appropriate credit to the original author(s) and the source, provide a link to the Creative Commons licence, and indicate if changes were made. The images or other third party material in this article are included in the article's Creative Commons licence, unless indicated otherwise in a credit line to the material. If material is not included in the article's Creative Commons licence and your intended use is not permitted by statutory regulation or exceeds the permitted use, you will need to obtain permission directly from the copyright holder. To view a copy of this licence, visit http://creativecommons.org/licenses/by/4.0/.

(C) The Author(s) 2020 